\title{
Application Status and Prospect of Pinus massoniana Sawdust as the Substrate for the Cultivation of Edible Fungi
}

\author{
Jinping Zhang, Menghao Du \\ Research Institute of Subtropical Forestry, Chinese Academy of Forestry, Hangzhou, China \\ Email: jinpingzhang@126.com
}

How to cite this paper: Zhang, J.P. and Du, M.H. (2017) Application Status and Prospect of Pinus massoniana Sawdust as the Substrate for the Cultivation of Edible Fungi. Food and Nutrition Sciences, 8, 1105-1113.

https://doi.org/10.4236/fns.2017.812082

Received: November 9, 2017

Accepted: December 12, 2017

Published: December 18, 2017

Copyright $\odot 2017$ by authors and Scientific Research Publishing Inc. This work is licensed under the Creative Commons Attribution International License (CC BY 4.0).

http://creativecommons.org/licenses/by/4.0/

\begin{abstract}
In this paper, the research status of using Pinus massoniana sawdust for the cultivation of edible fungi was analyzed and discussed. It was found that Pinus massoniana sawdust contained the material base of edible fungi with sufficient cellulose, hemicellulose and lignin and a small amount of nitrogen, phosphorus, potassium, calcium, magnesium, other mineral salts, and some vitamins for the growth of edible fungi. However, the Pinus massoniana wood contained a significant amount of terpenoids, which are detrimental to the growth of edible fungus and mycelial. The major and current detoxification methods for Pinus massoniana sawdust were analyzed, such as steaming method, distillation method, lime water immersion method, outdoor piling fermentation method, and chemical method. Their advantages and disadvantages were discussed and reviewed. Finally, the major issues in the application of Pinus massoniana sawdust as the cultivation substrate of edible fungi were analyzed, and the application prospect of Pinus massoniana sawdust as the substrate for the cultivation of edible fungi was also reviewed.
\end{abstract}

\section{Keywords}

Pinus massoniana Sawdust, Substrate, Edible Fungi, Terpenoids,

Detoxification Methods, Application Prospect

\section{Introduction}

Pinus massoniana Lamb, also known as pine, mountain pine, and fir pine, is a type of evergreen arbor and belongs to Pinus (Pinaceae). In the appropriate growth area, Pinus massoniana is distributed in 17 provinces (districts) in China, such as Guangdong, Guangxi, Yunnan, Fujian, Hunan, Hubei, Anhui, Sichuan, Guizhou, Henan, Shanxi, Jiangsu, Zhejiang, and Jiangxi etc. provinces [1]. 
The raw materials for the cultivation of edible fungi are predominately broad-leaved based sawdust. A lot of broad-leaved forest resources are consumed for this purpose each year. With the implementation of the national "natural forest protection project", forest cutting is strictly limited, which significantly limits the broad-leaved wood raw materials for the production of edible fungi. Therefore, to explore the use of softwood as edible fungus cultivation medium, the discovery and development of new sources of raw materials for the edible fungus cultivation have become a new topic in the edible fungus industry.

As the main tree species in southern China and the main timber in industrial, agriculture, construction, and daily life, the total amount of produced Pinus massoniana sawdust is up to hundreds of millions of tons annually, which is widely distributed in urban and rural areas and easy to be collected. Except that a small percentage of them are used as fuel, most of them are not utilized and treated as waste. The interfibrillar substance of Pinus massoniana contains a large number of impregnable compounds, such as resin, tannin, polyphenols, and turpentine. The high concentrations of these substances are detrimental to the growth of edible fungus mycelia. As a result, Pinus massoniana sawdust is rarely used as the cultivation medium for the edible fungi [2].

Currently, Pinus massoniana sawdust is mainly used for the substitute cultivation in the domestic and overseas. In other words, the treated Pinus massoniana sawdust is mixed with nutrients, growth promoters, and other additives for the cultivation of edible fungi. For example, Pinus massoniana treated with steaming and extensive exhaust method is used for the cultivation of shiitake mushroom. The produced mushroom is non-toxic and contents higher amounts of protein and phosphorus and lower amount of fat than mushroom cultivated with regular media. Therefore, it is a kind of high protein and phosphorus mushroom with low-fat content [3] [4]; Pinus massoniana fermented under natural piling conditions with added water for $8-12$ months can be used to cultivate various edible fungi, such as golden mushroom, Pleurotus eryngii, Pleurotus ostreatus, Auricularia polytricha, black fungus, and Hypsizygus marmoreus [5] [6] [7]. The pine sawdust treated with natural composting for two months was mixed with fresh pine sawdust and water evenly and then is composted for 60 days. After dried in the air, a total of $2 \%$ of magnesium sulfate, potassium dihydrogen phosphate, calcium carbonate, urea, and vitamin B was added and mixed evenly to afford the additive for the rapid degradation of pine resin. The additive with the amount of $5 \%$ is added to the dry pine sawdust and is fermented for five days at the temperature up to $50^{\circ} \mathrm{C}-52^{\circ} \mathrm{C}$. The result indicates that the content of pine resin in pine wood was reduced from 25.7 to $5.4 \mathrm{~g} / \mathrm{kg}$ [8]. The abovementioned steaming and extensive exhaust method has a high cost and incomplete removal of harmful substances, such as turpentine, tannin, and saponin. The method of natural fermentation requires a long time but results in incomplete removal of harmful substances. In addition, the above methods not only remove turpentine but also remove rosin that is beneficial to the growth of edible fungus mycelia. 


\section{The Material Base of Pinus massoniana Sawdust as the Substrate for the Cultivation of Edible Fungus}

The growth of edible fungi requires carbon, nitrogen, mineral elements, auxin, and other nutrients [9]. Pinus massoniana sawdust contains plenty cellulose, hemicellulose, lignin and a small amount of nitrogen, phosphorus, potassium, calcium, magnesium, and other minerals and some vitamins [10]. Overall, Pinus massoniana sawdust is rich with nutrients for the growth of edible fungi. According to the study by Yang Zhangqi, Pinus massoniana sawdust contained $45.409 \%$ of cellulose, $11.884 \%$ of pentose, $1.583 \%$ of cold water extract, $3.144 \%$ of hot water extract, $0.319 \%$ of ash, $28.389 \%$ of acid insoluble lignin, $14.056 \%$ extracts with $1 \% \mathrm{NaOH}, 9.692 \%$ of water, $2.592 \%$ extract with phenol alcohol [11]. This result is slightly different than the data reported by Pan Yuliang (Tab 1) but is similar to the result of $49.99 \%$ of carbon and $0.36 \%$ of ash from Chen Zhenxiong et al. [12]. The discrepancy in these data is related to the growth environment, tree age, trunk part, and measurement errors, and so on.

Pinus massoniana is one of the main species of pine trees and turpentine trees in China. Pine gum is mainly composed of rosin and turpentine oil. The main components of rosin are resin acids with a molecular formula of $\mathrm{C}_{20} \mathrm{H}_{3} \mathrm{O}_{2}$, as well as some of the fatty acids and non-acidic substances. Turpentine is a chain or cyclic olefin with a molecular formula of $\left(\mathrm{C}_{5} \mathrm{H}_{8}\right)_{n}$, namely a terpene mixture. The content of the terpene component varies greatly depending on the species, the quality of the raw material, and other conditions. As shown in Table 1, the main composition and content of Pinus massoniana gum in Guangxi with different tree ages ( $14-20$ years) and different tree diameters $(14-26 \mathrm{~cm})$ were the same. Trees with different ages contained the same main components with different contents, but there were no significant trends [13] [14] [15]. The content of a-pinene in Pinus massoniana turpentine oil was high, which has a boiling point of $155^{\circ} \mathrm{C}-156^{\circ} \mathrm{C}$. Except for the substances listed in Table 2, dipentene $\left(175^{\circ} \mathrm{C}\right.$ $\left.176^{\circ} \mathrm{C}\right)$, isoprene $\left(183^{\circ} \mathrm{C}-185^{\circ} \mathrm{C}\right)[13]$, limonene $(0.269 \%-0.377 \%)$, and sativene $(0.049 \%-0.106 \%)$ [16] were also identified in Pinus massoniana turpentine.

A experiment was conducted by Pan Yuliang, with addition of turpentine and rosin to the cultivation substrate of broad-leaved trees sawdust. It was found that shiitake mushroom mycelium was able to grow normally when the addition amount of rosin was $2 \%$ of the cultivation substrate. In contrast, when the addition amount of rosin was $0.1 \%$ of the cultivation substrate, the shiitake mushroom mycelium could not germinate and finally withered. Therefore, it is believed that the toxic substances which are harmful to mycelial growth are terpenes. If the content of terpenoids was reduced to $0.045 \%$ or less, there was no negative effect on the formation and quality of the infrared primordium and spore fruit of shiitake mushroom mycelium [3]. The experimental results demonstrated that terpenoids and th ir derivatives rather than rosin negatively affect the growth of shiitake mushroom mycelium [3] [4]. 
Table 1. the comparison of chemical compositions of Pinus massoniana sawdust with other commonly used woods for the cultivation of shiitake mushroom [3].

\begin{tabular}{ccc}
\hline Item & $\begin{array}{c}\text { Pinus massoniana } \\
\text { sawdust }\end{array}$ & $\begin{array}{c}\text { Other commonly used woods for } \\
\text { shiitake mushroom }\end{array}$ \\
\hline Carbon \% & 49.5 & $49.1-51.6$ \\
Hydrogen \% & 6.5 & $6.2-6.5$ \\
Oxygen \% & 43.2 & $41.2-43.5$ \\
Nitrogen \% & 0.8 & $0.8-1.2$ \\
Cellulose \% & 56.5 & $45-47$ \\
Lignin \% & 27.05 & $20-27$ \\
Poly pentose \% & 10.42 & $23-28$ \\
Acid soluble matter \% & 2.40 & $0.45-1.5$ \\
Material soluble in hot water \% & 2.90 & $2.30-3.14$ \\
Terpenoids \% & 0.4 & 0 \\
Tannin \% & 0.3 & $2-3$ \\
\hline
\end{tabular}

Table 2. Main components and content of Pinus massoniana gum [13] [14] [15] [16].

\begin{tabular}{cccc}
\hline Main components of rosin & Content \% & Main components of turpentine oil & Content \% \\
\hline Pimaric acid & $0.32-0.73$ & $\alpha$-Pinene & $47.07-78.07$ \\
Elliotinoic acid & $7.01-10.47$ & Camphene & $0.87-1.50$ \\
Isopimaric acid & $1.43-1.86$ & $\beta$-Pinene & $2.30-10.92$ \\
L-pimaric acid & $40.77-53.58$ & Myrcene & $0.05-0.54$ \\
Dehydroabietic acid & $0.13-0.39$ & $\Delta$-Camphene & $0.33-1.68$ \\
Abietic acid & $7.25-17.71$ & Longifolene & $5.82-27.25$ \\
Neoabietic acid & $9.46-17.92$ & Caryophyllene & $2.78-5.15$ \\
\hline
\end{tabular}

According to the above analysis, Pinus massoniana sawdust contains a high content of cellulose and slightly lower content of polydextrose and tannic acid with terpenoids. As long as appropriate measures are taken to remove or reduce the content of harmful substances and to add essential nutrients for mycelium growth and reproduction, Pinus massoniana sawdust can be used as the cultivation substrate for edible fungi.

\section{Research Status of Pretreatment Technology for the Preparation of Cultivation Substrate for Edible Fungi from Pinus massoniana Wood}

Pinus massoniana sawdust containing terpenoids is detrimental to the growth of edible fungus mycelium and cannot be directly used as for the substitute cultivation of edible fungi. Terpene is a transparent, colorless, flowing liquid with a special aroma, high volatility, and high boiling point $\left(150^{\circ} \mathrm{C}-185^{\circ} \mathrm{C}\right)$. It is insoluble in water but soluble in alcohol, benzene, carbon disulfide, and gasoline. 
Many domestic researchers majored in edible fungi investigated the detoxification technology of pine wood sawdust by utilizing the volatile property of terpenoids and put forward various methods, such as steaming exhaust method, distillation method, lime water immersion method, outdoor stacking fermentation method, and chemical method [17]. However, these methods are tedious, energy-consuming, or significant nutrient loss in the process treatment, which is unfavorable for the improvement of cultivation yield, thus limiting the application and promotion of these methods in the production of edible fungi.

\subsection{Steaming Method}

The Pinus massoniana sawdust is placed into a cauldron containing $1 \%-2 \%$ lime water. The resulting mixture is heated to boiling and constantly stirred with a stick. After boiling for 2 hours, the mixture is rinsed with water [18]. The disadvantages of this method are the incomplete removal of terpene compounds and excessive consumption of energy and water [3] [19]. The steaming and extensive exhaust method is the use of steaming the submerged pine sawdust to remove the harmful substances, which is carried out by normal pressure steaming or high-pressure steaming along with extensive exhaust during the steaming process [3] [19] [20]. Normal pressure steaming requires long treating time, and the removal of the terpene substances is incomplete. Although high-pressure steaming requires a short time, this process has a highly demanding equipment, complicated procedure, and high cost.

\subsection{Distillation Method}

Distillation method is the removal of the harmful ingredients in Pinus massoniana sawdust by using distillation. Specifically, after placing a bottomless barrel into a pot, water is added to the pot until the distance of the water level to the board is $10 \mathrm{~cm}$. Then, sawdust is added and compacted, followed by drilling a few vent holes with a stick. Next, the cover of the pot is closed to prevent leakage, and water is filled to full. The cooling tube and the upper part of the cooler are connected to the pot. The bottom of the cooler extends to the outside of the barrel and is connected to the oil and water separator. After distillation with intensive heating for about $2-3$ hours, oil is removed and collected. The distillation is completed after $4-5$ hours, and the cooled sawdust is ready for use [17]. In another process, a vent pipe is installed in the bottom of a large steel barrel with a diameter of 1 meter from the exterior to the interior of the barrel. A screen is placed on the top of vent pipe located inside the barrel, and a layer of glass fiber bag is added on the screen. The wet Pinus massoniana sawdust containing $60 \%$ moisture is evenly put on the glass fiber bag with a thickness of $4-7$ $\mathrm{cm}$. Then, steam is supplied to the barrel through the vent pipe. When the steam is passed through the Pinus massoniana sawdust, more sawdust is added. The addition process is repeated until the bucket is full. After the completed the addition of sawdust, the barrel is closed with one vent hole is open. The supply of 
steam is stopped after 6 hours [21]. The distillation method is similar to above mentioned. However, this method can only extract a small amount of turpentine because the boiling point of turpentine is above $155^{\circ} \mathrm{C}$. As a result, Pinus massoniana sawdust treated with distillation still have the inhibitory effect on the growth of mycelia.

\subsection{Lime Water Immersion Method}

Lime water immersion method involves the immersion of Pinus massoniana sawdust in a certain concentration of lime water for a period of time and the removal of the sawdust from the lime water, followed by rinsing the treated sawdust with water until the rinsing water reaches transparency and $\mathrm{pH}$ of 7 7.5. Finally, the water is drain, and the sawdust is sundried for use [22] [18]. The principle of this method is based on the saponification of pine resin with alkali to form soluble soap that can be removed by water washing [23]. The disadvantage of this method is the incomplete elimination of the terpenoids and alkaloids.

\subsection{Turmeric Fermentation Method}

In Japan, the detoxication method for the treatment of Pinus massoniana sawdust is long-term water accumulation. The pile of Pinus massoniana sawdust is generally a rectangular heap or square heap. Water is sprayed when piling the sawdust. The moisture content of sawdust is maintained about $60 \%$ by spraying water periodically. The piling typically takes $6-12$ months. The pile is turned over 2 - 3 times so that the pile is homogeneous. The Pinus massoniana sawdust treated with this piling method not only can be used to cultivate golden mushroom, Pleurotus ostreatus, Pleurotus eryngii and other edible mushrooms but also can be used to cultivate Pleurotus ostreatus, hair fungus, black fungus and other conventional varieties [5]. The day before the piling, the Pinus massoniana sawdust is pre-wetted. When piling, the layers of Pinus massoniana sawdust and aqueous urea solution are added alternately. The lower layer of Pinus massoniana sawdust is not watered. The middle and upper layers of Pinus massoniana sawdust are added a small amount and a large amount of water, respectively. The exudation of water around the pile indicates the amount of added water is sufficient. Upon the completion of the piling, the pile is covered with plastic film and a layer of grass curtain to retain heat and moisture. After piled for three months, the pile is turned over one time. Before the use, the moisture content and $\mathrm{pH}$ of the treated sawdust are adjusted. The Pinus massoniana sawdust treated with this method can be used to cultivate golden mushroom [24]. The Pinus massoniana sawdust mixed with $1 \%-2 \%$ of the lime water is fermented naturally for $30 \mathrm{~d}$. After drying, the sawdust is stored for use. Before use, the sawdust should be dried for one more day [25]. The fermentation cycle of Pinus massoniana sawdust can be reduced to $20 \mathrm{~d}$ by adding some dry horse manure, $0.5 \%$ urea and $0.1 \%$ of the fermentation agent. The successfully fermented ma- 
terial is soft without aroma. It has a moisture content of about $60 \%$ so that additional water is not needed when mixing for cultivation [18]. Inorganic nutrient minerals (magnesium sulfate, potassium dihydrogen phosphate, and calcium carbonate) that can promote the growth of microorganisms and organic nitrogen sources (urea, vitamins, and vitamins B, etc.) in an amount of dry sawdust weight of $0.1 \%-1 \%$ are added to the microorganisms isolated from the natural fermentation of Pinus massoniana sawdust to prepare the additives after evenly mixed. The prepared additive is added to dry Pinus massoniana sawdust in a ratio of 5\%. After natural fermentation for five days, the content of pine resin is reduced from $2.57 \%$ to $0.54 \%$ [8]. The drawback of this method is this process degrades both harmful turpentine and helpful rosin that promotes the growth of edible fungus. Since the content of turpentine treated with this process has not been reported yet, it is hard to determine whether the treated Pinus massoniana sawdust is suitable for the cultivation of edible fungus mycelium.

\subsection{Chemical Method}

Given the volatile and reductive properties of the terpene species in Pinus massoniana sawdust, Wu Xueqian et al. developed a process for quick removal of terpenoids in the pine wood by adding the chemical substance called "fungus booster and mildew killer" with both oxidizing and antimildew activities. In the meantime, nutrients that can promote the quick growth of the mycelium are added to reinforce the nutrients in the culture [4]. The procedure and mechanism of this process, and the ingredients of the chemical substance "fungus booster and mildew killer" for removing terpenoids have not been reported so far in the literature.

The above-mentioned treatment methods have certain effects in practical applications, of which the fermentation method is simple and practical, and has been used most frequently. However, the degradation and metabolic pathways of cellulose, hemicellulose, lignin, rosin, and turpentine in Pinus massoniana wood are not clear because there are no reports in this research field.

\section{Major Issues in the Application of Pinus massoniana Sawdust as the Cultivation Substrate of Edible Fungi}

Although some progress and achievements have been achieved in the application of Pinus massoniana sawdust as the cultivation substrate of edible fungi, there is a lack of systematic research on the application of Pinus massoniana for the cultivation of edible fungi. As a matter of fact, there are still various issues to be addressed, such as material base of Pinus massoniana sawdust as the cultivation substrate of edible fungus, the analysis of component that is detrimental to mycelial growth of edible fungus, pretreatment methods for the elimination of the harmful substances and degradation of macromolecular organic matters, mechanism of degradation of macromolecular organic matters, screening of substrate formula, strain selection of edible fungus, composition analysis of edible 
fungus, evaluation of nutritional value, demonstration for food safety, and industrial cultivation.

\section{Application Prospect of Pinus massoniana Sawdust for the Cultivation of Edible Fungi}

With the continuous improvement of technology and scientific research methods, the studies on the application of Pinus massoniana sawdust as the cultivation substrate for edible fungi will become deeper and broader. In the meantime, the research focus will be shifted from the traditional simple pretreatment, feasibility study, and screening of substrate formula to investigation on the material basis of the application of Pinus massoniana sawdust as the cultivation substrate of edible fungi, identification of the harmful substances and their contents on the growth of mushroom mycelial, screening of materials that can degrade harmful substances and macromolecular organic matters, elucidation of the conversion process and degradation mechanism of harmful substances and macromolecular organic matters, and construction of technical system consisting of physical, chemical, and biological methods for rapid and efficient applications of Pinus massoniana sawdust.

\section{Acknowledgements}

The authors are grateful for the financial support from National Key R\&D Program of China, Grant No. 2017YFD0601002.

\section{References}

[1] Zhou, Z.X. (2011) Chinese Pinus massoniana. China Forestry Press, Beijing, 14.

[2] Deng, L.J. and Fu, J.P. (1996) A Brief Study on the Cultivation of Oyster Mushroom Using Pine Sawdust. Edible Fungi, 5, 18.

[3] Pan, Y.L. (1982) Studies on the Utilization of Pinus massoniana Sawdust for the Cultivation of Shiitake Mushroom. Guangxi Agricultural Science, 29-32.

[4] Wu, X.Q., Wei, H.L., Wu, Y.K., et al. (2008) New Efficient Antimildew Technology for the Cultivation of Shiitake Mushrooms Using Pine and Fir Sawdust. Zhejiang Edible Fungi, 16, 30-33.

[5] Qiu, G.G. (2003) Piling Technology for the Treatment of Japan Pine and Fir Sawdust. Edible Fungi or Fungi, No. 3, 39.

[6] Chen, Q. and Huang, C.Y. (2013) Current Situation of Japanese Edible Fungus Industry. Edible Fungi of China, 32, 67-69, 72.

[7] Fan, L.L. (2006) Technology for the Cultivation of Hair Fungus Using Pine and Fir Wood Chips. Guangxi Agricultural Sciences, 37.

[8] Wu, Q.P., Chen, S.Y., Que, S.H., et al. (2003) Method for Rapid Degradation of Pine Resin in Pine Sawdust. Patent No. CN200310112187.6, Application Date: 2003.11.19.

[9] Si, J.P., Xu, Y.K., Fu, G.W., et al. (2003) Experimental Study on the Cultivation of Shiitake Mushrooms with Bags of Magnolia officinalis Sawdust. Zhejiang Forestry Science and Technology, 23, 16-18. 
[10] Qin, B.S. and Qin, Y.R. (2010) Status and Prospect of New Cultivation Substrate for Edible Fungi. Chinese Agricultural Science Bulletin, 26, 223-228.

[11] Yang, Z.Q. (2012) Sdudies on Genetic Variation of Timber Chemical Composition of Pinus masso. Journal of Fujian College of Forestry, 32, 188-192.

[12] Chen, Z.X., Liu, X.P. and Xiong, Z.P. (2011) Carbon Content and Caloric Value Analysis of Pinus massoniana. Central South Forest Inventory and Planning, 30, 54-58.

[13] An, N. and Ding, G.J. (2012) Studies on the Chemical Composition of Pine Resin of Pinus massoniana in Guangxi. Journal of Central South University of Forestry and Technology, 32, 59-62.

[14] An, N., Ding, G.J., Jia, H.Y., et al. (2015) Study on the Difference on the Fat Production and Pine Resin Composition of Pinus massoniana with Different Trunk Diameters. Journal of Central South University of Forestry, 35, 42-45.

[15] Yang, Z.Q. (2014) Analysis of the Fat Production and Rosin Components of Pinus massoniana with Different Ages. Forestry Science, 50, 147-151.

[16] Liu, Q., Wei, Y., Fan, H., et al. (2016) Variation and Correlation Analysis of Chemical Components of Pine Resin in Pinus massoniana. Forestry Science Research, 29, 324-330.

[17] Wang, Y. (2000) Cultivation of Shiitake Mushroom using Pine and Fir Sawdust. Panzhihua Science \& Technology and Information, 53.

[18] Wei, L., Zhang, Z., Jiang, T., Li, Y. and Xue, J. (2006) Study and Application of Technology Utilizing Softwood Sawdust for the Cultivation of Edible Fungi. Agriculture and Technology, 26, 58-59, 72.

[19] Hon, D.N.-S. and Shiraishi, N. (1991) Wood and Cellulosic Chemistry. Marcel Dekker Inc.

[20] Norimoto, M. and Gric, J. (1989) Wood Bending using Microwave Heating. Journal of Microwave Power and Electromagnet Energy, 24, 203-212. https://doi.org/10.1080/08327823.1989.11688095

[21] Xu, L. and Sui, J. (2006) A Method of Removing Pine Resin in Pine Sawdust to Prepare Culture Material for the Cultivation of Mushroom. Patent Number CN200610164472.6.

[22] Yan, Z. (1995) Cultivation of Edible Fungi with Pine Sawdust. Zhejiang Edible Fungi, 28.

[23] Zhu, Z. (1993) Wetland. Guangdong Science and Technology Press.

[24] Wu, J. and Wang, J. (2014) Cultivation of Mushroom using Pine and Fir Sawdust. Edible Fungi, No. 4, 46-47.

[25] Xiao, S., Liu, Y.G., Weng, H., et al. (2007) Investigation on the Cultivation of Abalone Mushroom using Pine and Fir Sawdust. Edible Fungus, No. 2, 28. 